\title{
Hierarchical Topic Aggregation for Geospatial Applications through Processing Extensions in Message Brokers
}

\author{
Josef Spillner \\ josef.spillner@zhaw.ch \\ Zurich University of Applied Sciences \\ Winterthur, Switzerland
}

\begin{abstract}
Publish/subscribe (pub/sub) message brokers are typically operated with multiple tenants and multiple topics per tenant. Messages sent by a producer to a topic are received by consumers who subscribed to the same topic. In many applications, topic hierarchies are a desired property. This is especially the case for geospatial applications in which regions are composed of smaller-scale regions. However, most message brokers do not support hierarchies natively. HTABroker demonstrates how a light-weight processing function deployed to an extensible message broker overcomes this limitation for several geospatial index systems.
\end{abstract}

\section{ACM Reference Format:}

Josef Spillner. 2020. Hierarchical Topic Aggregation for Geospatial Applications through Processing Extensions in Message Brokers. In 21st International Middleware Conference Demos and Posters (Middleware '20 Demos and Posters), December 7-11, 2020, Delft, Netherlands. ACM, New York, NY, USA, 2 pages. https://doi.org/10.1145/3429358. 3429370

\section{Problem Statement}

Digitalised societies and democratic participation of e-citizens, smart cities and regions, as well as autonomous driving and navigation are application fields which benefit from strong geospatial computing. In this computing paradigm, data is tagged with location information, and therefore subject to implicit relations such as is-nearby, is-neighbour-of, or consists-of. The paradigm implies aggregation functionality. For instance, each state of a country may report case

Permission to make digital or hard copies of all or part of this work for personal or classroom use is granted without fee provided that copies are not made or distributed for profit or commercial advantage and that copies bear this notice and the full citation on the first page. Copyrights for components of this work owned by others than the author(s) must be honored. Abstracting with credit is permitted. To copy otherwise, or republish, to post on servers or to redistribute to lists, requires prior specific permission and/or a fee. Request permissions from permissions@acm.org. Middleware '20 Demos and Posters, December 7-11, 2020, Delft, Netherlands (c) 2020 Copyright held by the owner/author(s). Publication rights licensed to ACM.

ACM ISBN 978-1-4503-8202-1/20/12 ..\$15.00

https://doi.org/10.1145/3429358.3429370 numbers in case of an epidemy, and the country-wide numbers are calculated as the sum of the cases per state. Building such system for scalable use in real-time requires a powerful messaging infrastructure, and at the core of it, distributed message brokers that support propagation relations.

Existing brokers available to software engineers today do not possess these capabilities. They implement many features around reliable, scalable and low-latency messaging [2], but are not sufficiently treating geospatial information as first-class citizen. This contrasts database management systems that have possessed such capabilities for many years (e.g. PostGIS, MongoDB, Redis, Tile38) [1]. As a generalisation, the support for general hierarchical topics, channels or queues is also of concern. While hierarchical naming is typically supported to enable filtering (e.g. main. *), propagation and aggregation across these hierarchies is not. Table 1 summarises the capabilities of a selection of existing brokers.

Table 1. Message brokers overview; *: denotes extensibility

\begin{tabular}{lll}
\hline Broker & Geospatial & Hierarchical \\
\hline \hline NATS & none & only multi-sub via regexp \\
\hline RabbitMQ & none & only multi-sub via regexp \\
\hline Kafka & none & only multi-sub via list/regexp \\
\hline VerneMQ & none & none \\
\hline Pulsar ${ }^{*}$ & none & only multi-sub via list/regexp \\
\hline
\end{tabular}

\section{Research and Technical Approach}

The solution consists of (1) defining appropriate hierarchical geospatial models, (2) defining propagation and aggregation operations, and (3) implementing the solution with userdefined processing logic (functions) atop existing message brokers to benefit from the existing scalability and resilience.

\subsection{Models}

HTABroker supports several geospatial models. Apart from computational models whose data points are calculated on demand, further data-driven models are expressed in treestructured data files. The current version of HTABroker integrates with four models, also called index systems: 
- Computational: Geohash. Each frame between two longitude/latitude pairs is represented by one or multiple geohashes which are alphanumerical codes. With arbitrary precision $n \geq 1$, this results in $36^{n}$ entries.

- Data-driven: NUTS. The Nomenclature des unités territoriales statistiques is used in the EU and associated countries. It subdivides countries into regions and districts. Apart from Local Administrative Units (LAUs), there are currently around 1500 entries defined.

- Data-driven: Communes. More localised than NUTS, the Swiss Communes model exemplifies the relation between a country, its subdivisions (cantons, districts) and municipalities. It contains 2200 municipalities.

- Hybrid: H3. Used by Uber navigation, regions are divided into hexagons. It contains around 8700 entries.

\subsection{Messaging}

Apart from simple publish/subscribe message delivery, the hierarchical topic aggregation foresees three underlying propagation directions that are configurable per application.

- Upward propagation. An event is produced in a subentity (e.g. geo region) and propagated to its superentity. For instance, the registration of a foreigner as state resident leads to changed country-wide statistics.

- Downward propagation. For instance, a new countrywide alert is replicated on municipal apps that mix hierarchy levels for the benefit of the users.

- Sideward propagation. Events are propagated to neighbouring entities. For instance, a traffic jam in a city district affects rerouting decisions in nearby places.

Fig. 1 shows an example of the three propagation types with subdivisions of a country in the NUTS model, with producers $(\mathrm{P})$ and consumers $(\mathrm{C})$ of messages.

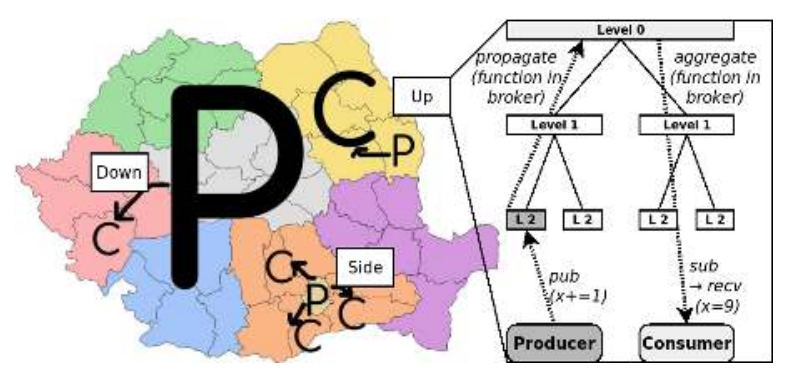

Figure 1. Propagation directions in a NUTS scheme

\subsection{Software and Integration}

HTABroker is implemented as a Python library, CLI and set of aggregation functions, allowing for both local eventing simulation and attachment to a Pulsar broker into which the HTA aggregation functions are deployed programmatically along with propagation in/out links and data-driven models as JSON files. The CLI includes convenient features such as fully qualifying shortcut identifiers by recursively scanning the hierarchies. Listing 1 shows an example of a simple subscribe-publish sequence using downward propagation.

Listing 1. Sample transcript; ))): prompt; \#: debug message

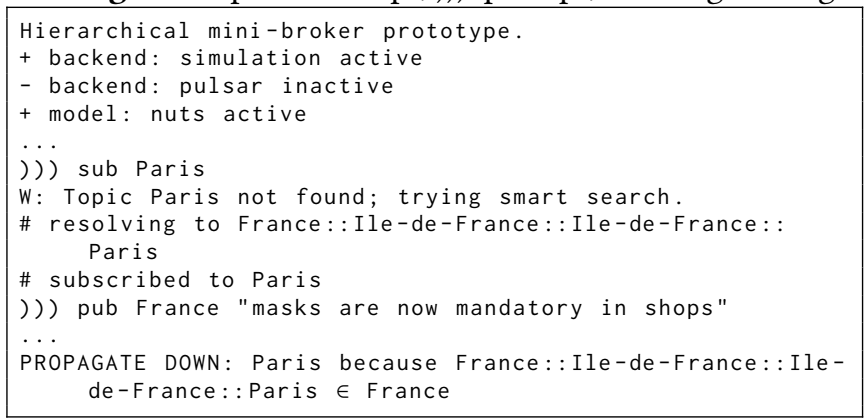

\section{Related Work}

Pallatella et al. investigated aggregation of MQTT topics using multi-topic subscription patterns which is a closely related concept but without any geospatial bindings [5]. For wide-area IoT, Livaja et al. proposed geospatial pub/sub conceptually, but do not introduce a working solution [4]. Kassab et al. built a prototype for real-time emergency that processes GPS locations, without propagation directions [3].

\section{Conclusions}

HTABroker is a versatile real-time messaging middleware for geospatial applications. The software is available as open source at https://github.com/serviceprototypinglab/htabroker.

\section{Acknowledgments}

This work is supported by a DIZH Fellowship under grant Smart Cities and Regions Services Enablement (SCReSE).

\section{References}

[1] Dominik Bartoszewski, Adam Piórkowski, and Michal Lupa. 2019. The Comparison of Processing Efficiency of Spatial Data for PostGIS and MongoDB Databases. In 15th International Conference, BDAS 2019, Ustroń, Poland, May 28-31, 2019, Proceedings (CCIS, Vol. 1018). Springer, 291-302. https://doi.org/10.1007/978-3-030-19093-4_22

[2] Philippe Dobbelaere and Kyumars Sheykh Esmaili. 2017. Kafka versus RabbitMQ: A comparative study of two industry reference publish/subscribe implementations: Industry Paper. In Proc. 11th ACM Intl. Conf. Distributed and Event-based Systems, DEBS 2017, Barcelona, Spain, fune 19-23, 2017. ACM, 227-238. https://doi.org/10.1145/3093742.3093908

[3] Ala' Kassab, Steve Liang, and Yang Gao. 2010. Real-time notification and improved situational awareness in fire emergencies using geospatialbased publish/subscribe. Int. 7. Appl. Earth Obs. Geoinformation 12, 6 (2010), 431-438. https://doi.org/10.1016/j.jag.2010.04.001

[4] Ivan Livaja, Dejan Skvorc, and Kresimir Pripuzic. 2017. Geospatial publish / subscribe systems for the Internet of Things. In 25th International Conference on Software, Telecommunications and Computer Networks, SoftCOM 2017, Split, Croatia, September 21-23, 2017. IEEE, 1-8. https://doi.org/10.23919/SOFTCOM.2017.8115542

[5] Maria Rita Palattella, Ridha Soua, André Stemper, and Thomas Engel. 2018. Aggregation of MQTT Topics over Integrated Satellite-Terrestrial Networks. SIGMETRICS Perform. Evaluation Rev. 46, 3 (2018), 96-97. 